Yan Senlin*

\title{
Study of Chaos Control of a Dual-Ring Erbium-Doped Fiber Laser Using Parameter Method
}

https://doi.org/10.1515/joc-2018-0021

Received February 06, 2018; accepted August 01, 2018

\begin{abstract}
This paper aims to study single-parameter and dual-parameter chaos control of a dual-ring erbium-doped fiber laser. We present the control model to pressure chaotic behavior of the laser to a periodic state or multi-periodic states by introducing a periodic mutational signal to adjust the losses of dual ring. When the loss of single ring is shifted between two different levels, the laser behaviors can be deduced to two single-period states, while two rings can emit cyclic pulses. We find a control-locking regime and discuss its frequency characteristic of the laser. When another signal is taken to control to perform on the loss, the laser can be deduced to show a period- 2 state, a period-3 state, period-4 state and other multi-periodic states. And we find dual-dynamic controlled regimes, in which, one ring of the laser can be produced to show a periodic state while another ring presents a period- 3 state. We find also a dualperiod regime or a period- 4 regime. When both the losses of dual ring are adjusted by shifting between different levels, the laser can produce a period-4, a period- 6 and other multi-periodic states. The result indicates that chaos control of a dual-ring erbium-doped fiber laser can be realized using the single-parameter and dual-parameter methods.
\end{abstract}

Keywords: laser, chaos, control

\section{Introduction}

Chaotic dynamic has the unique characteristic of being sensitive to its starting condition and external effects. Chaotic motion is random and its output signal is similar to white noise. It would be very difficult for people to forecast long-term chaotic behavior. Since chaos-control method of "OGY" was presented in the twentieth century [1], many chaos-control technique have been presented to control chaotic variety $[2,3]$. Currently, chaotic lasers are being used in many fields and people pay attention to chaos control of lasers. People obtained chaos control of lasers to stabilize the laser at a periodic state by the current modulation and the optical feedback, and so on $[3,4]$. However, we see little of reports about control of chaotic dual-ring erbium-doped fiber lasers. In the twentieth century, erbium-doped fiber is used as a novel laser source and optical amplifier in optical fiber communications and optical fiber sensors [5-12]. Chaotic lasers are used to secure communication, random signal generator and radar [7, 8]. A dual-ring fiber laser arises chaotic output using optical mutual injection of the two ring lasers $[7,8,13]$. In this paper, novel chaos-control methods of single-parameter and dual-parameter methods are presented using a periodic mutational signal to adjust the loss. There are many fascinating physical phenomena, and other types of lasers are hard to see. The result is helpful to study the chaos control of lasers $[14,15]$.

\section{Model}

The physical model of dual-ring erbium-doped fiber laser can be described by coupling the two single-ring erbiumdoped fiber lasers. After considering the control planshown in figure 1 , the laser normalized field and the normalized ion number are described by the coupling rate equation as follows [3, 4, 7-10]:

$$
\begin{gathered}
\frac{d}{d t} E_{a}=-k_{a} \times\left[1-\mu_{a} \times s_{a}\left(2 \pi f_{a} t\right)\right] \times\left(E_{a}+\eta_{0} E_{b}\right)+g_{a} E_{a} D_{a} \\
\frac{d}{d t} E_{b}=-k_{b} \times\left[1-\mu_{b} \times s_{b}\left(2 \pi f_{b} t\right) \times\left(E_{b}-\eta_{0} E_{a}\right)+g_{b} E_{b} D_{b}\right.
\end{gathered}
$$

$$
\frac{d}{d t} D_{a}=-\left[1+I_{p a}+\left|E_{a}\right|^{2}\right\} D_{a}+I_{p a}-1
$$

*Corresponding author: Yan Senlin, School of Electronic Engineering, Nanjing XiaoZhuang University, Nanjing, China, E-mail: senlinyan@163.com 


$$
\frac{d}{d t} D_{b}=-\left[1+I_{p b}+\left|E_{b}\right|^{2}\right\} D_{b}+I_{p b}-1
$$

where subscripts $a$ and $b$ stand for the ring "a" and ring "b" of the laser, $I_{p}$ indicates the laser pump. $E$ indicates the laser normalized field. $D$ indicates the normalized ion number, $k$ indicates the loss coefficient, $\eta_{0}$ indicates the coupling coefficient of the coupler and $g$ indicates the gain coefficient. The laser can generate chaotic optical pulses or some nonlinear dynamic behaviors when the dual-ring coupling level reaches a high value. In eqs.(1) and (2), the term $-\mu_{a, b} \times s_{a, b}\left(2 \pi f_{a, b} t\right)$ is introduced to realize chaos-control operation by a loss modulator shifting the loss of two rings. $s_{a, b}$ stands for a periodic mutational signal, $\mu_{a, b}$ is the shifting depth and $f_{a, b}$ is the shifting frequency of signal. This additional degree of freedom as a control term being induced to operate the loss will result in nonlinear effects, and these functions will affect laser behavior. We find that the laser output will be deduced to cyclic pulses or other multi-cycle pulses, while chaos control of dual-ring erbium-doped fiber laser will be able to be obtained effectively using mutational parameter method.

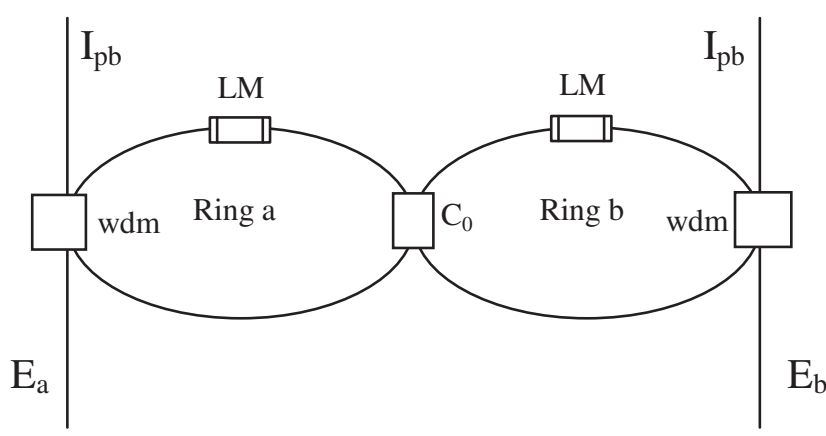

Figure 1: A schematic of chaos control, where $I_{p a}$ and $I_{p b}$ are the pump light, $C_{0}$ is the coupler, wdm is the wavelength division multiplexer and $E_{a}$ and $E_{b}$ are the optical fields from ring "a" and ring "b", respectively. LM is the loss modulator.

\section{Results of single-parameter control of the ring $b$}

In our numerical simulations, the laser parameters taken as normalized values $[3,4,7,8,13]$ are as follows: $I_{p a}=I_{p b}=4, \quad k_{a}=\mathrm{k}_{\mathrm{b}}=1,000, \quad \eta_{0}=0.2, \quad g_{a}=4,800$, $g_{b}=10,500$. When control-control operation does not carry out, Figure 2 gives nonlinear dynamic behavior of two rings of the laser, where Figure 2(a) shows chaotic attractor and Figure 2(b) shows dual-ring emitting optical fields. We find chaotic behavior being very sensitive to its starting condition and showing different trajectories when the initial conditions are taken as different values.

First, we discuss the results of single-parameter chaos control of laser when the loss of the ring $b$ is shifted between two levels. Let the control parameter $\mu_{b}=0.4$ and $s_{b}$ switches on the value 1 or the value zero and when the frequency is $f_{b}=11 \mathrm{kHz}$; chaos control is performed on the laser after $2 \mathrm{~ms}$ for all control processes. Figure 3 shows the laser motion in chaos-control processes, where chaotic laser is controlled to stabilize in a cycle-4 state after $5 \mathrm{~ms}$. Figure 3(a) gives the laser becoming of a cycle-4 trajectory, Figure 3(b) shows dual-ring output variation along with some undamped relaxation oscillation from $2 \mathrm{~ms}$ to $4 \mathrm{~ms}$ and Figure 3(c) stands for two stable cycle- 3 states shown in two rings. The above results indicate that we have succeeded to obtain controlling this chaotic dual-ring erbium-doped fiber laser.

When the frequency is taken as $f_{b}=10 \mathrm{kHz}$, the laser can be deduced to cycle- 6 states, while the dual ring emits cycle-6 states shown in Figure 4. Figure 4(a) shows the laser dynamics behavior with a cycle- 6 trajectory and Figure 4(b) shows dual-ring output varying with cycle-6 pulses.

When the frequency is reduced to $f_{b}=9 \mathrm{kHz}$, the laser can be controlled to stabilize in cycle- 3 states, while the dual ring emits cycle-3 pules shown in Figures 5(a) and (b).

We reduce the frequency to $f_{b}=7 \mathrm{kHz}$; this control performance results in cycle- 8 states shown in the laser while the dual-ring can emit cycle-8 pules presented at Figures 6(a) and (b).

When we add the frequency to $f_{b}=16 \mathrm{kHz}$, the control performance can result in that the laser can become of other cycle- 6 states while the dual-ring emits cycle- 6 pulses shown in Figure 7. Figure 7(a) shows the laser dynamics with a cycle-6 trajectory and Figure $7(\mathrm{~b})$ shows dual-ring output varying with cycle-6 pulses. Here, coupling effect of two ring dominates laser behavior, leading to the same period- 6 state.

When the frequency is increased to $f_{b}=16.8 \mathrm{kHz}$, the control performance causes the laser to cycle- 4 states while the dual ring shows cycle-4 pulses in Figures 8(a), (b) and (c).

When the frequency is increased to $f_{b}=17.5 \mathrm{MHz}$, laser motion can be brought tolock at stable cyclic states while the dual ring output emits cyclic pulses shown in Figure 9. Figure 9(a) presents the laser dynamics 


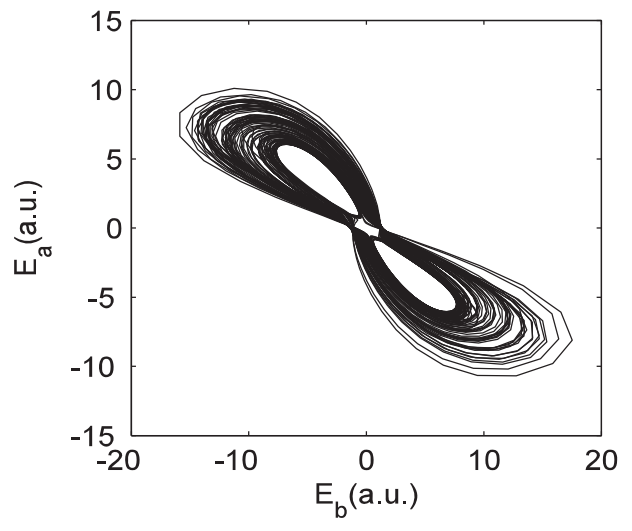

(a) typical chaotic attractor
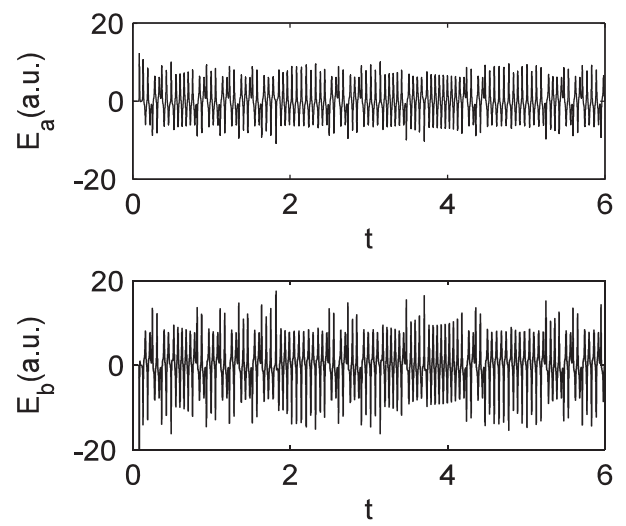

(b) dual-ring output

Figure 2: Chaotic dynamics: (a) typical chaotic attractor, (b) dual-ring output.

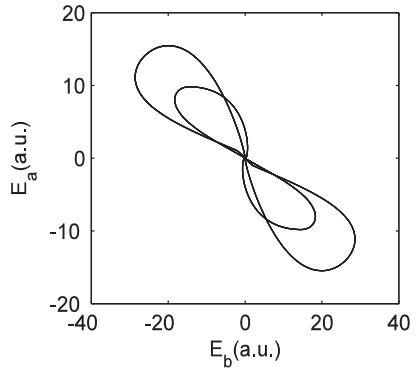

(a) cycle-4 trajectory
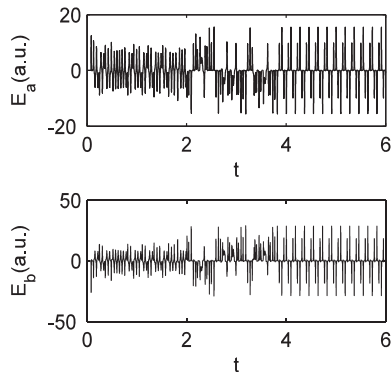

(b) dual-ring output
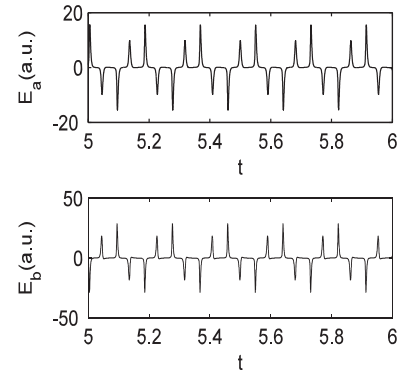

(c) two cycle-4 waveforms

Figure 3: The laser shows cycle-4 states: (a) cycle-4 trajectory, (b) dual-ring output and (c) two cycle-4 waveforms.

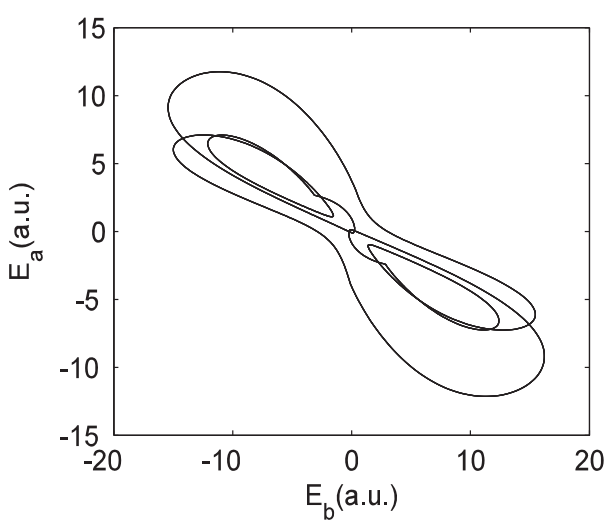

(a) cycle-6 trajectory
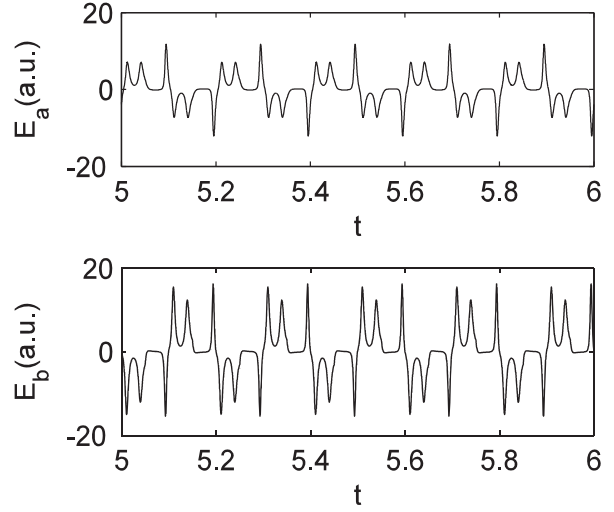

(b) cycle-6 pulses from two rings

Figure 4: The laser becoming of cycle-6 states: (a) cycle- 6 trajectory and (b) cycle-6 pulses from two rings.

behavior becoming of a cyclic trajectory and Figure 4(b) shows a dual ring emitting a periodic wave varying with cyclic pulses at an oscillation frequency of $17.5 \mathrm{kHz}$. We find a control-locking region that shows periodic regional distribution from $17.5 \mathrm{kHz}$ to $24 \mathrm{kHz}$. In this region, the laser realizes to stabilize at some cyclic states, while the two rings are locking at the shifting frequencies. These results indicate that we have succeeded to realize to control the chaotic dual-ring erbium-doped fiber laser. 


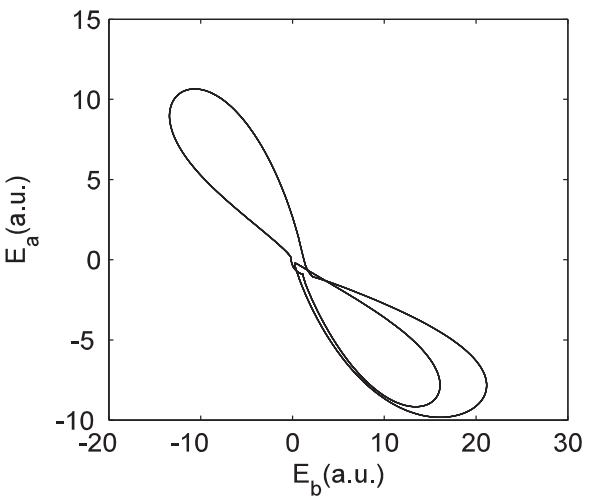

(a) cycle-3 trajectory
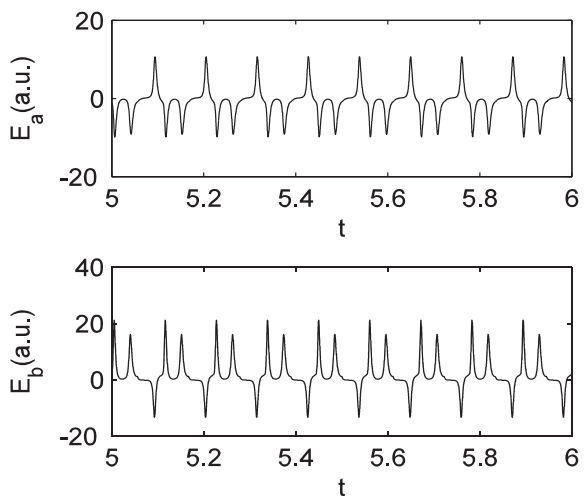

(b) two ring show cycle-3 behaviors

Figure 5: The dual ring at cycle-3 states: (a) cycle-3 trajectory and (b) two rings show cycle-3 behaviors.

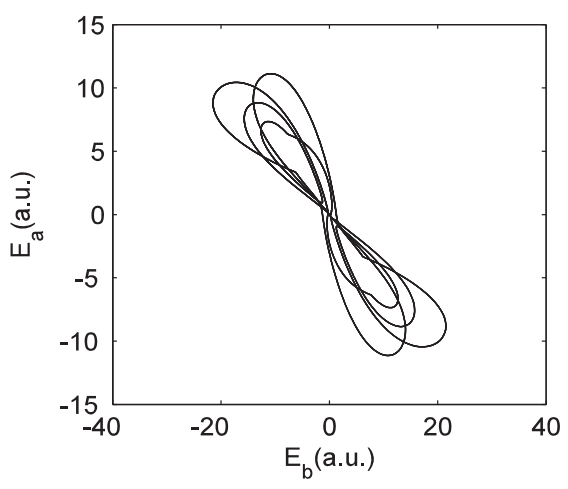

(a) cycle-8 trajectory
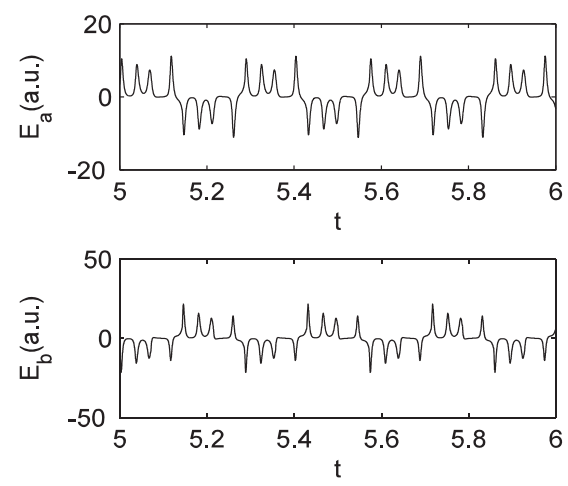

(b) two ring show cycle- 8 behaviors

Figure 6: The dual-ring becoming of cycle- 8 states: (a) cycle- 8 trajectory and (b) two rings show cycle- 8 behaviors.

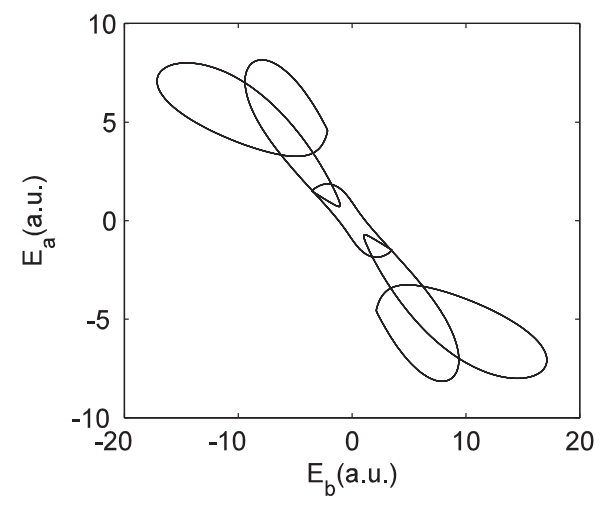

(a) other cycle-6 trajectory
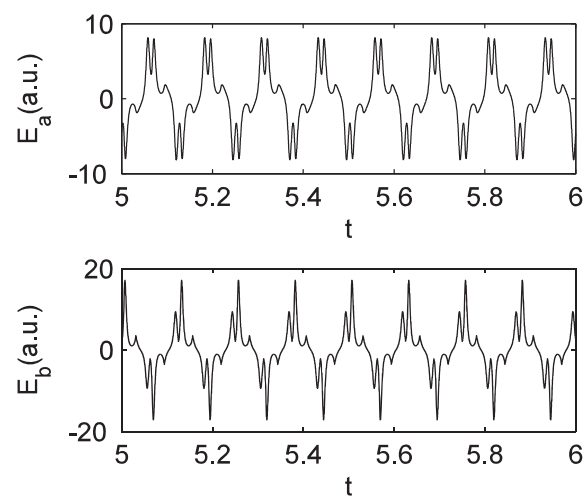

(b) two ring output cycle-6 waveforms

Figure 7: The dual ring becoming of other cycle- 6 states: (a) Other cycle-6 trajectory and (b) two-ring output cycle- 6 waveforms.

When we increase the frequency to $f_{b}=26 \mathrm{kHz}$ again, the control result is shown in Figure 10, where the laser becomes of cycle-2 states. When the frequency is increased to $f_{b}=27 \mathrm{kHz}$, the laser is deduced to cycle-3 states shown in Figure 11.
We find a dual-period region and a cycle-4 region. The dualperiod region distributes between $35 \mathrm{kHz}$ and $36 \mathrm{kHz}$ and the period-4 region distributes between $40 \mathrm{kHz}$ and $41 \mathrm{kHz}$. We find also another dual-period region that distributes from 44 $\mathrm{kHz}$ to $53 \mathrm{kHz}$. 


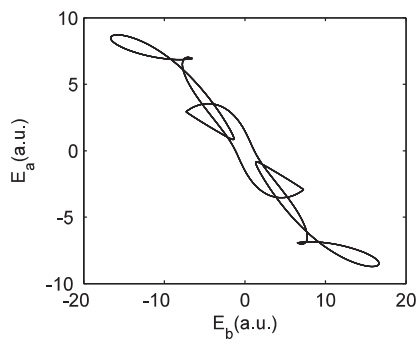

(a) other cycle-4 trajectory
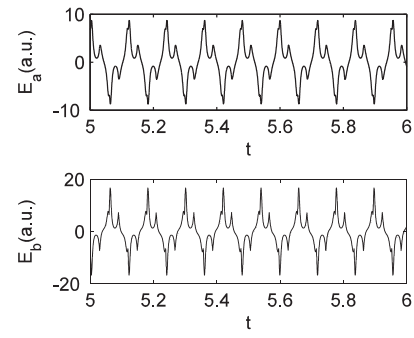

(b) cycle-4 waveforms
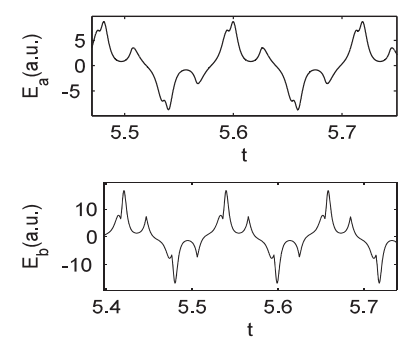

(c) zoomed fig.4 (b)

Figure 8: The dual ring becoming of other cycle-4 states: (a) other cycle-4 trajectory, (b) cycle-4 waveforms and (c) zoomed Figure 4(b).

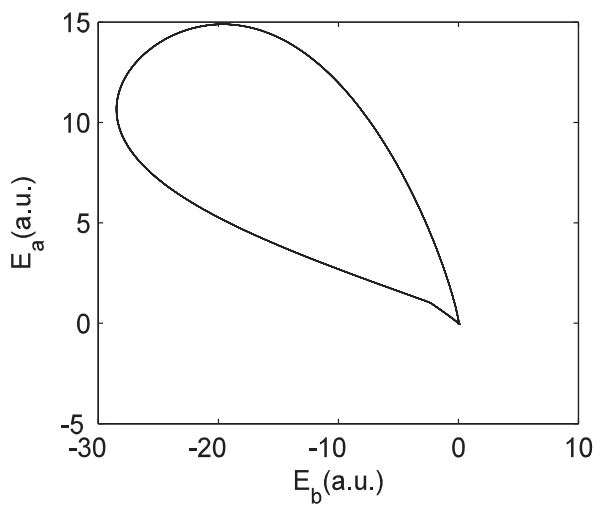

(a) a cyclic trajectory
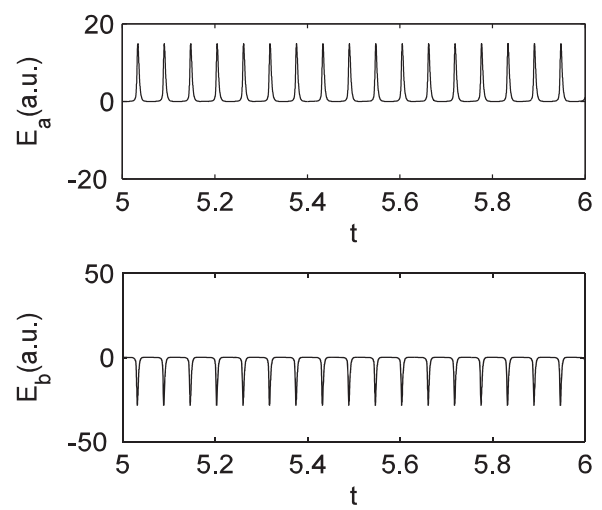

(b) dual-ring emits cyclic pluses

Figure 9: The dual-ring locking at cyclic states: (a) a cyclic trajectory and (b) dual-ring emitting cyclic pluses.

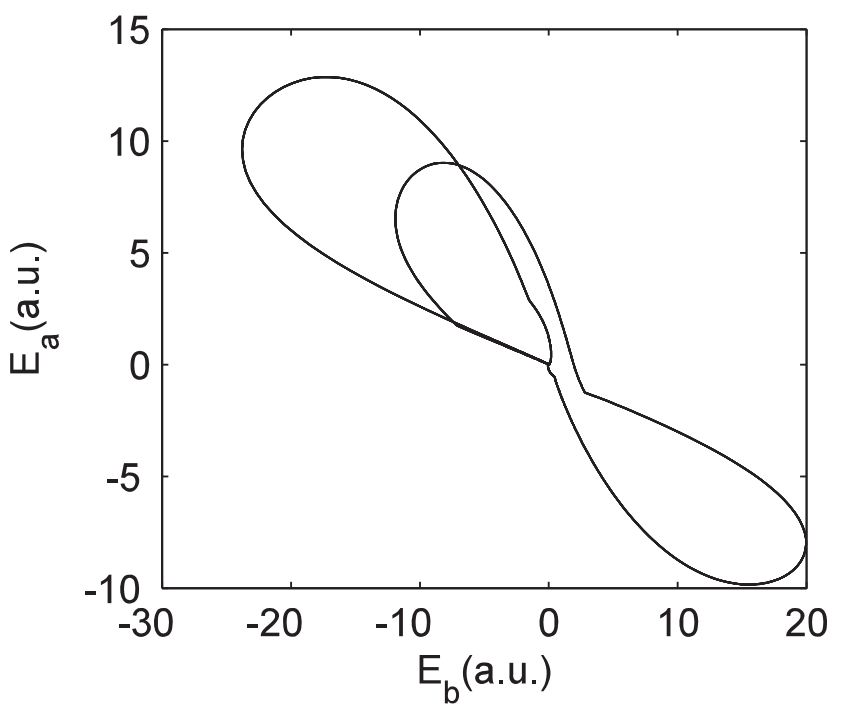

Figure 10: Cycle-2 trajectory.

When we perform on the laser using another signal with a high-frequency and a low-shifting depth taken as $f_{b}=65$ $\mathrm{kHz}$ and $\mu_{b}=0.2$, the two rings can be deduced to show

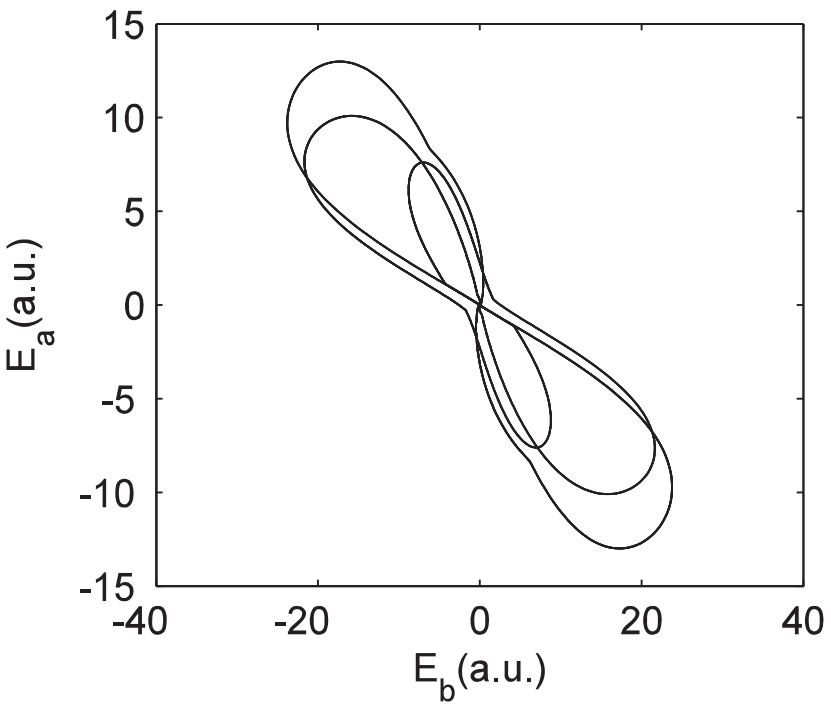

Figure 11: Cycle-3 trajectory.

two different states, where the ring "a" can produce cyclic pulses and the ring "b" can arise cycle-3 pulses shown in Figure 12. We find such chaos-controlled dual-dynamic region from $55 \mathrm{kHz}$ to $70 \mathrm{kHz}$. 

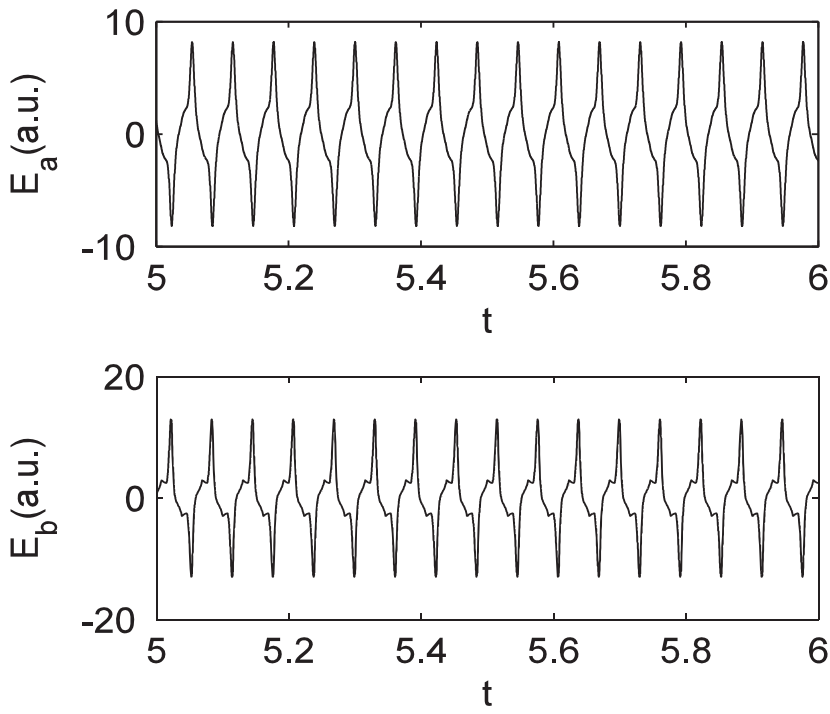

Figure 12: The dual-ring becomes of different states.

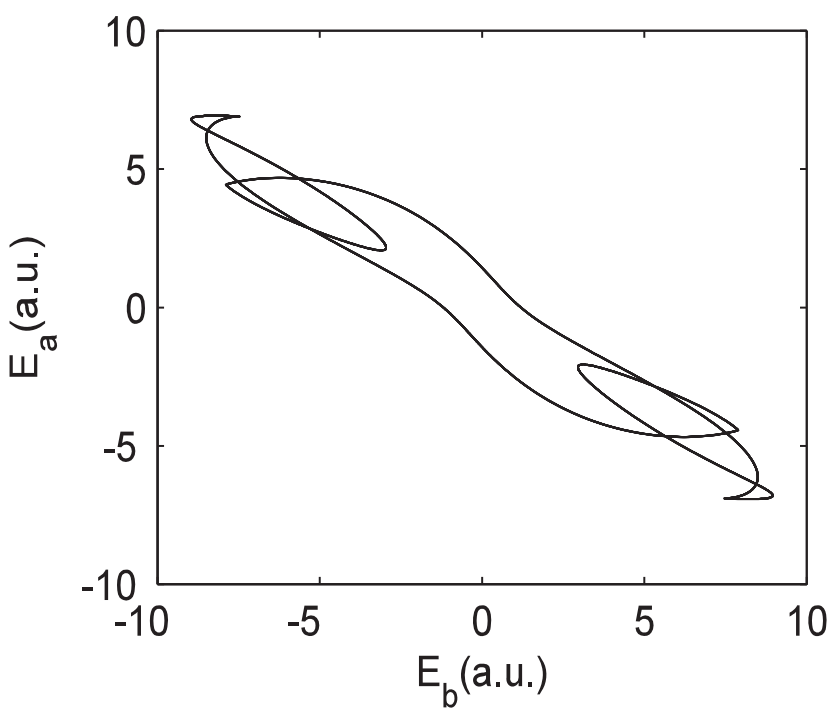

Figure 13: Cycle-6 trajectory.

When the control parameter is taken as $\mu_{b}=0.2$ and $f_{b}=18 \mathrm{kHz}$, the two rings can raise two different states, where the ring "a" shows a cycle-3 behavior and the ring "b" shows a cycle-5 behavior shown in Figure 13. The gain can affect laser oscillation and the gain coefficient differences in the two rings. Here, the gains of two ring dominate laser behavior to some extent, so the laser ring "b" is being excited at a cycle- 5 oscillation by the control signal while the laser ring "a" shows a cycle-3 oscillation. When we operate on the laser using other signals at a frequency $f_{b}=18 \mathrm{kHz}$ and different shifting depths taken as the values $\mu_{b}$ between 0.1 and 0.008 , the two rings of the laser can be deduced to lock at periodic states at a frequency of $18 \mathrm{kHz}$.

\section{Results of dual-parameter control of the dual ring}

We study the dual-parameter control and make chaos-control performance on the losses of the dual ring of the laser, in which the loss of the ring "a" is shifted by a shifting signal with the parameters being $f_{a}=20 \mathrm{kHz}, \mu_{a}=0.1$ and $s_{a}$ between values 1 and 0 while the loss of the ring " $b$ " is

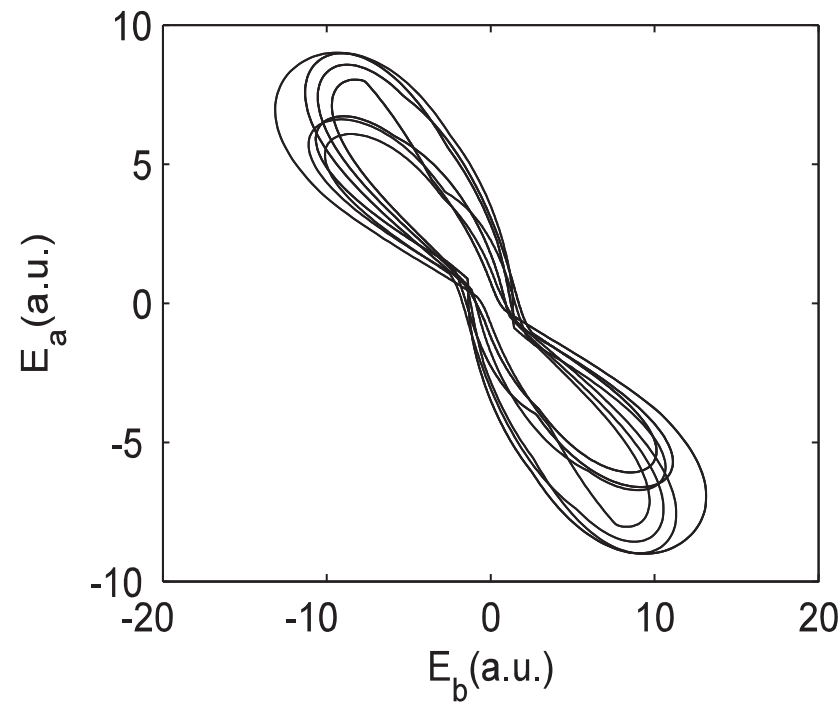

Figure 14: The laser has cycle-6 trajectory.

shifted by a shifting signal with the parameters being $f_{b}=18 \mathrm{kHz}, \mu_{b}=0.1$ and $s_{b}$ between values 1 and 0 . Figure 14 shows that the laser motion can be controlled to stabilize in cycle-6 states. The result can indicate that chaotic dualring erbium-doped fiber laser can be controlled.

When the control parameters are taken as $f_{a}=15 \mathrm{kHz}$, $\mu_{a}=0.1, f_{b}=18 \mathrm{kHz}, \mu_{b}=0.1$ and $s_{a, b}$ is between values 1 and 0 , the laser behavior can be deduced to show cycle- 6 states shown in Figure 15.

\section{Conclusion}

The single-parameter and dual-parameter chaos-control method of the dual-ring erbium-doped fiber laser are 


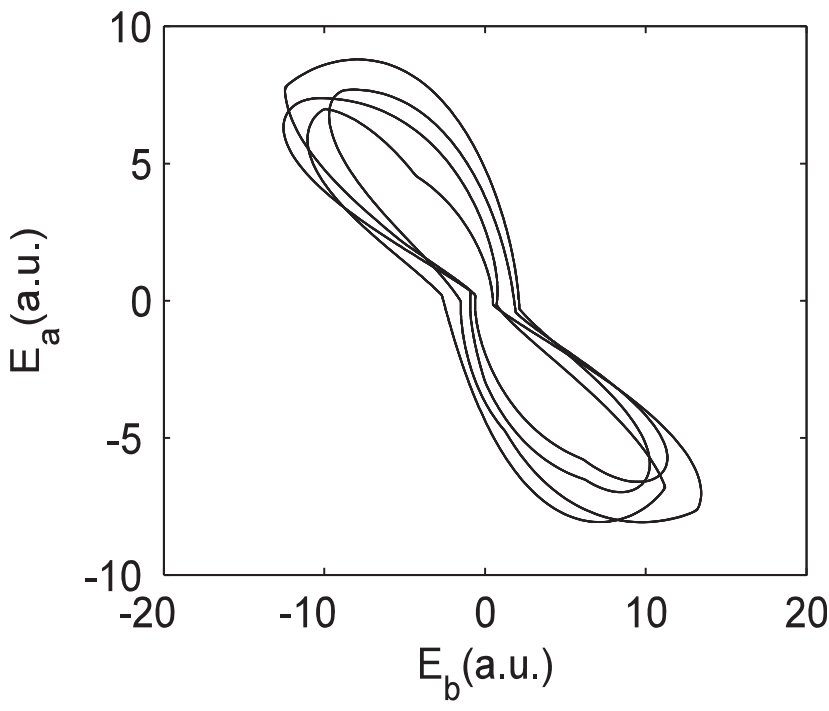

Figure 15: The laser has cycle-6 trajectory.

studied in this paper. The laser can be controlled to singlecycle states or some multiple-cycle states. Compared to the previous report $[2-5,7,9,13]$, now the laser is controlled by mutational excitation of square wave signal as control signal substituting sine signal, and dual-signal is used to obtain thereliable reazlization of chaos control of laser. Dual-parameter control is easier than single-parameter control to produce high-dimensional periodic state. Those improvements and innovations have been made to control this chaotic laser, so the controlled-locking region, dualcycle region, other multi-cycle region, such as high-dimensional periodic states, and dual-dynamical behaviors are produced to be found. Some fantastic phase space orbits will arouse our interest in the study of the laser. We will study this chaos-control method in experiment and give a suggestion for researchers interested in chaos control to find these controlled phenomena in experiment. Reference literature $[2,5,7,9,13]$ will provide some good references for us to control chaos experimentally. The result is very helpful for our study of chaos-control, dual-ring erbiumdoped fiber laser and other lasers. Considering its application prospect in the field of control and laser, we feel that further chaos-control research in theory and experiment is needed to discover new physical phenomena, propose new control methods and promote the development of new laser chaos-control technology.

\section{References}

[1] Ott E, Grebogi C, Yorke JA. Controlling chaos. Phys Rev Lett. 1990;64:1196-99.

[2] Ming D, Peter KC. Analysis of Er-doped fiber laser stability by suppressing relaxation oscillation. IEEE J Photonic Technol Lett. 1996;8:1151-53.

[3] Yan SL. Study on the method of controlling chaos in an Erdoped fiber dual-ring laser via external optical injection and shifting optical feedback light. Chaos. 2007;17:013106-06.

[4] Yan SL. Control of chaos in an injection multi-quantum-well laser via shifting or modulating the injection light. J Mod Opt. 2009;56:539-47.

[5] Williams OL, Ojalvo JG, Roy R. Fast intra-cavity polarization dynamics of an erbium-doped fiber ring laser: inclusion of stochastic effects. Phys Rev A. 1997;55:2376-85.

[6] Sancheez F, Stephan G. General analysis of instabilities in erbium-doped fiber laser. Phys Rev E. 1996;53:2110-22.

[7] Luo LG, Chu PL, Liu HF. 1-GHz optical communication system using chaos in erbium-doped fiber lasers. IEEE J Quantum Electron. 2000;12:269-71.

[8] Wang R, Shen K. Synchronization of chaotic systems modulated by another chaotic system in an erbium-doped fiber dualring laser system. IEEE J Quantum Electron. 2001;37:960-63.

[9] Sola J, Martín JC, Álvarez JM. Nonlinear response of a unidirectional erbium-doped fiber ring laser to sinusoidally modulated pump power. Opt Commun. 2002;212:359-69.

[10] Alexander NP, Alexander VK, Yuri OB. Dynamics of an erbiumdoped fiber laser with pump modulation: theory and experiment. J Opt Soc Am B. 2005;22:2107-14.

[11] Fan L, Wu Z, Deng T, Wu J, Tang X, Chen J, et al. Subharmonic microwave modulation stabilization of tunable photonic microwave generated by period-one nonlinear dynamics of an optically injected semiconductor laser. IEEE/OSA J Lightwave Technol. 2014;32:4058-64.

[12] Lindley BS, Schwartz IB. Modeling synchronization in networks of delay-coupled fiber ring lasers. Optics Express. 2011;19:24460-67.

[13] Luo LG, Tee TJ, Chu PL. Chaotic behavior in erbium-doped fiber ring lasers. Opt Soc Am B. 1998;15:972-78.

[14] Guo B. 2D noncarbon materials-based nonlinear optical devices for ultrafast photonics. COL. 2018;16:020004-35.

[15] Guo B, Lyu Q, Yao Y, Wang P. Direct generation of dip-type sidebands from WS2 mode-locked fiber laser. Opt Mater Express. 2016;6:2475-887. 